\title{
AN ITERATION METHOD FOR CALCULATION WITH LAURENT SERIES*
}

\author{
BY \\ H. A. RADEMACHER AND I. J. SCHOENBERG \\ University of Pennsylvania and Ballistic Research Laboratories, Aberdeen Proving Ground
}

Introduction. The power series is a basic concept of Analysis which is of fundamental importance from the theoretical as well as from the computational point of view. The theoretical importance of power series springs from the fact that it represents any analytic function in the neighborhood of a regular point. The reason for its practical importance is the ease with which implicitly defined functions, by finite relations or differential equations, may be expanded in power series by the so-called method of undetermined coefficients, known and used since the dawn of mathematical analysis.

Laurent series play a definitely minor role as compared to power series. One reason is the more complicated nature of the connection between the sum of the series and its coefficients. Another reason, dependent on the first, is the difficulty of calculations with Laurent series.

The purpose of this paper is to describe a method whereby rational or algebraic operations with Laurent series may be performed with high accuracy at the expense of a reasonable amount of labor. A general approximation method to empirical data: developed by one of us, ${ }^{1}$ required the very accurate reciprocation of certain Lauren 1 series. This problem of reciprocation of Laurent series was the starting point of ous investigation. Our method for solving this particular problem turned out to be identi. cal with a method of reciprocation of finite matrices already investigated by $\mathrm{H}$. Hotelling. ${ }^{2}$ We finally point out that our method of computation with Lauren series extends to computations with trigonometric series provided these series con verge absolutely.

1. Newton's algorithm and statement of the problem. Let

$$
f(x) \equiv a_{0} x^{m}+a_{1} x^{m-1}+\cdots+a_{m}=0, \quad\left(a_{0} \neq 0\right),
$$

be an algebraic equation with numerical real or complex coefficients. If $x$ is a simpl root of this equation, then very close approximations to $x$ may be readily computer by Newton's iterative algorithm represented by the recurrence relation

$$
x_{n+1}=x_{n}-\frac{f\left(x_{n}\right)}{f^{\prime}\left(x_{n}\right)} .
$$

The reason for the fast convergence of $x_{n}$ towards $x$ is as follows: Expanding the right

* Received Nov. 6, 1945.

${ }^{1}$ I. J. Schoenberg, Contributions to the problem of approximation of equidistant data by analytic, func. tions, Part A, Quart. Appl. Math.

${ }^{2}$ H. Hotelling, Some new methods in matrix calculation, Ann. Math. Statist. 14, 1-34 (1943), espe cially p. 14, and Further points in matrix calculation and simultaneous equations, Ann. Math. Statist. 14 440-441 (1943). 
hand side in a power series in $x_{n}-x$, in the neighborhood of the simple root $x$, we find that (2) may be written as

$$
x_{n+1}-x=c_{2}\left(x_{n}-x\right)^{2}+c_{3}\left(x_{n}-x\right)^{3}+\cdots,
$$

with coefficients $c_{\nu}$ depending only on the root $x$. Because there is no linear term in $x_{n}-x$ on the right-hand side we find that from a certain point on the error $x_{n+1}-x$ is of the order of magnitude of the square of the previous error $x_{n}-x$. From this stage on, each step will approximately double the number of correct decimal places of the previous approximation $x_{n}$. This type of rapid convergence is sometimes referred to as "quadratic convergence."

Let us notice that the iterative process (2) requires a division, by $f^{\prime}\left(x_{n}\right)$, at each step of the process. This is a serious handicap in computing with machines which do not perform the operation of division, as for example the standard punch-card machines. This division is likewise a handicap if we wish to extend the process to the realm of matrices where division is a difficult numerical operation.

We propose to modify Newton's algorithm (2) so as to require only the operations of addition, subtraction and multiplication in its performance. It will then be shown how the modified Newton algorithm allows us to carry out numerically rational as well as algebraic operations on Laurent series. The most general numerical problem whose solution is facilitated by our method may be formulated as follows.

Problem. Let

$$
f(w, z) \equiv a_{0}(z) w^{m}+a_{1}(z) w^{m-1}+\cdots+a_{m}(z)=0
$$

be an equation with the following properties:

1. The coefficients $a_{v}(z)$ are all regular and uniform functions of $z$ in the ring

2. We have

$$
R: \quad r_{1}<|z|<r_{2} \text {. }
$$

$$
a_{0}(z) \neq 0 \text { in } R .
$$

3. The discriminant $D(z)$ of (4) satisfies

$$
D(z) \neq 0 \text { in } R
$$

so that the equation (4) has no critical point in $R$. Let now $w=w(z)$ be a branch of a solution of (4) which is necessarily regular in $R$ but need not be uniform in $R$. Given the numerical values of the Laurent expansions of the coefficients $a_{\nu}(z)$, the problem is to find the values of the coefficients of the Laurent expansions of w(z).

Remarks. 1. The difficulty of this problem is due to its being concerned with Laurent series rather than ordinary power series. Indeed, if everything else is unchanged, we replace, in its formulation, the ring (5) by the circle $|z|<r_{2}$, then all Laurent series mentioned become power series, in which case the power series expansion of the branch $w=w(z)$ may be obtained by the method of undetermined coeffcients (see the first paragraph of our Introduction).

2. We did not require the branch $w=w(z)$ to be uniform in $R$. However, we do not restrict our problem by assuming $w(z)$ to be uniform in $R$. Indeed, if $w(z)$ returns to its values after $k$ turns in $R, k>1$, we change variable by setting

$$
z=\zeta^{k} \text {. }
$$


Our equation (4) thereby becomes

$$
f\left(w, \zeta^{k}\right)=0
$$

and the branch $w(z)$ becomes uniform in the corresponding ring in the $\zeta$-plane. If we can determine its uniform Laurent series

$$
w(z)=\sum_{-\infty}^{\infty} \omega_{n} \zeta^{n}
$$

we also have its algebraic expansion

$$
w(z)=\sum_{-\infty}^{\infty} \omega_{n} z^{n / k} .
$$

3. Even the case $m=1$ is far from trivial. Thus

$$
a_{0}(z) w-1=0
$$

amounts to the important problem of the reciprocation of a given Laurent series.

2. The modification of Newton's algorithm. We return in this section to the case of the ordinary algebraic equation (1). We now impose the restriction that

$$
f(x) \text { has only simple zeros. }
$$

This condition implies that the polynomials $f(x), f^{\prime}(x)$ have no common divisors and that we can therefore determine uniquely, by rational operations alone, two polynomials $\phi(x)$ and $\psi(x)$ satisfying the identity

$$
f(x) \phi(x)+f^{\prime}(x) \psi(x) \equiv 1,
$$

and such that the degrees of $\phi$ and $\psi$ do not exceed $m-2$ and $m-1$, respectively. The coefficients of $\phi(x), \psi(x)$ are rational functions of the coefficients $a_{v}$. For later reference it is important to remark that the coefficients of $\psi(x)$ may be written as a quotient of polynomials in $a$, divided by the discriminant $D$ of the polynomial $f(x)$. Indeed, the coefficients of $\phi$ and $\psi$ are determined, in view of (9), by a system of linear equations whose determinant is precisely the discriminant $D$ of $f(x)$. This procedure leads to explicit expressions of $\psi$ and $D$ in determinant form. Thus for $m=3$ we obtain

$$
\psi(x)=\frac{1}{D}\left|\begin{array}{ccccc}
x^{2} & x & 1 & 0 & 0 \\
3 a_{0} & 0 & 0 & a_{0} & 0 \\
2 a_{1} & 3 a_{0} & 0 & a_{1} & a_{0} \\
a_{2} & 2 a_{1} & 3 a_{0} & a_{2} & a_{1} \\
0 & a_{2} & 2 a_{1} & a_{3} & a_{2}
\end{array}\right|, \quad D=\left|\begin{array}{ccccc}
3 a_{0} & 0 & 0 & a_{0} & 0 \\
2 a_{1} & 3 a_{0} & 0 & a_{1} & a_{0} \\
a_{2} & 2 a_{1} & 3 a_{0} & a_{2} & a_{1} \\
0 & a_{2} & 2 a_{1} & a_{3} & a_{2} \\
0 & 0 & a_{2} & 0 & a_{3}
\end{array}\right| .
$$

This expression, which generalizes to any value of $m$, indeed shows that the coeffcients of $\psi(x)$ have the common denominator $D$ if regarded as rational functions of the $a_{\nu}$ 's.

Now we modify Newton's algorithm (2) to its new form ${ }^{3}$

${ }^{3}$ We learn from a note by J. S. Frame, Remarks on a variation of Newton's method, Amer. Math. Monthly, 52, 212-214 (1945), that precisely the same modification of Newton's algorithm has already been used since 1942 by H. Schwerdtfeger, of the University of Adelaide, South Australia, for the numerical solution of ordinary algebraic and transcendental equations. 


$$
x_{n+1}=x_{n}-f\left(x_{n}\right) \psi\left(x_{n}\right) .
$$

Setting

$$
F(x) \equiv x-f(x) \psi(x)
$$

we may write (11) as

$$
x_{n+1}=F\left(x_{n}\right) .
$$

On comparing (2) and its modification (11) we see that the division required by (2), at each step of the process, is not present in (11). Now we want to show that the algorithm $\left(11^{\prime}\right)$ also enjoys the property of (2) of producing fast convergence towards the zeros of $f(x)$. Indeed, let $x$ be a root of (1),

$$
f(x)=0,
$$

and let us expand $F\left(x_{n}\right)$ about the point $x_{n}=x$. Writing for convenience $f^{(\nu)}(x)=f^{(v)}$, $\psi^{(v)}(x)=\psi^{(p)}$, we have by Taylor's formula

hence

$$
\begin{aligned}
& f\left(x_{n}\right)=f+f^{\prime}\left(x_{n}-x\right)+\frac{1}{2} f^{\prime \prime}\left(x_{n}-x\right)^{2}+\cdots, \\
& \psi\left(x_{n}\right)=\psi+\psi^{\prime}\left(x_{n}-x\right)+\frac{1}{2} \psi^{\prime \prime}\left(x_{n}-x\right)^{2}+\cdots,
\end{aligned}
$$

$f\left(x_{n}\right) \psi\left(x_{n}\right)=f \psi+\left(f \psi^{\prime}+f^{\prime} \psi\right)\left(x_{n}-x\right)+\frac{1}{2}\left(f \psi^{\prime \prime}+2 f^{\prime} \psi^{\prime}+f^{\prime \prime} \psi\right)\left(x_{n}-x\right)^{2}+\cdots$.

By (9) and (13) we have $f=0, f^{\prime} \psi=1$ and therefore

$$
F\left(x_{n}\right)-x=-\frac{1}{2}\left(2 f^{\prime} \psi^{\prime}+f^{\prime \prime} \psi\right)\left(x_{n}-x\right)^{2}+\cdots .
$$

This shows that we may write our relation (11) in the form

$$
x_{n+1}-x=b_{2}(x)\left(x_{n}-x\right)^{2}+b_{3}(x)\left(x_{n}-x\right)^{3}+\cdots+b_{2 m-1}(x)\left(x_{n}-x\right)^{2 m-1},
$$

where the $b_{v}(x)$ are polynomials in $x$ with coefficients which are polynomials in $a_{v}$ divided by the common denominator $D$.

Again the missing linear term in $x_{n}-x$, on the right-hand side of (14), shows that if $x_{0}$ is sufficiently close to $x$, then the algorithm (11) will insure that $x_{n} \rightarrow x$ with quadratic convergence.

An important special case of (1) is the equation

$$
a x^{m}-1=0, \quad(a \neq 0) .
$$

The identity

$$
(-1)\left(a x^{m}-1\right)+\frac{x}{m}\left(\max ^{m-1}\right)=1
$$

shows that in this case

$$
\psi(x)=\frac{1}{m} x .
$$

The relation (11) now becomes

$$
x_{n+1}=x_{n}+\frac{1}{m} x_{n}\left(1-a x_{n}^{m}\right) .
$$

In particular if $m=1,(15)$ reduces to

$$
a x-1=0
$$


when (16) becomes

$$
x_{n+1}=x_{n}+x_{n}\left(1-a x_{n}\right) .
$$

3. The reciprocation of matrices. The advantage of the modified, or division-free, Newton algorithm (11) appears in connection with matrix calculations. In recent years $\mathrm{H}$. Hotelling has recommended the following procedure of finding the reciprocal $X=A^{-1}$ of a given numerical non-singular matrix

$$
A=\left\|\alpha_{i j}\right\| \quad(i, j=1, \cdots, m) .
$$

Obtain in some way, e.g. by the so-called Gauss, or Doolittle, process a good approximation $X_{0}$ to $A^{-1}$. Then improve this approximation by the recurrence relation

$$
X_{n+1}=X_{n}+X_{n}\left(I-A X_{n}\right) .
$$

In the case of $m=1$ this relation is identical with (18).

In studying the convergence of $X_{n}$ towards $X=A^{-1}$ Hotelling metrizes the space of real $m \times m$ matrices by means of the absolute value or norm

$$
N(A)=\sqrt{\sum_{i, j} \alpha_{i j}^{2}}
$$

which enjoys the following properties

$$
N(A+B) \leqq N(A)+N(B), \quad N(A B) \leqq N(A) N(B) .
$$

By means of these inequalities Hotelling derived an estimate of $N\left(X_{n}-X\right)$ which was improved by A. T. Lonseth as follows: ${ }^{4}$

Inequality of Hotelling and Lonseth. Let $X_{0}$ be an approximation to $X=A^{-1}$ such that

$$
N\left(I-A X_{0}\right)=k<1 .
$$

Starting with $X_{0}$ we obtain the sequence $X_{n}$ by (20). Then

$$
N\left(X_{n}-X\right) \leqq N\left(X_{0}\right) \cdot k^{2^{n}} \cdot(1-k)^{-1} .
$$

This interesting result shows in particular that the inequality (23) is sufficient to insure the convergence of the process.

Our generalizations (16) and (11) of the recurrence relation (18) suggest similar iterative procedures for the solution of non-linear algebraic matrix equations. We prefer, however, to pass on to a discussion of calculations with Laurent series.

4. Calculations with Laurent series. Let

$$
a(z)=\sum_{-\infty}^{\infty} \alpha_{n} z^{n}, \quad\left(r_{1}<|z|<r_{2}\right),
$$

be a Laurent series converging in the ring (5). There is no inherent restriction of the generality of the Problem formulated in our Introduction if we assume that the ring $R$ contains the unit-circle $|z|=1$, i.e.

4 See Hotelling's second note already mentioned. 


$$
r_{1}<1<r_{2}
$$

An advantage of this normalization is that it implies that $\alpha_{n} \rightarrow 0$ exponentially as $n \rightarrow+\infty$ or $n \rightarrow-\infty$, insuring that the sequence $\left\{\alpha_{n}\right\}$ is "finite" to a fixed number of decimal places.

The relation (25) sets up a one-one correspondence

$$
a(z) \sim\left\{\alpha_{n}\right\}
$$

between functions $a(z)$ uniform and regular in $R$ and sequences $\left\{\alpha_{n}\right\}$ for which the series (25) converges in $R$. To the function $a(z) \equiv 1$ corresponds the unit-sequence

$$
I: \quad \alpha_{0}=1, \alpha_{n}=0 \text { if } n \neq 0 .
$$

This correspondence may be interpreted as an isomorphism concerning the operations of addition, subtraction, multiplication and multiplication by a scalar. Indeed, if

$$
b(z)=\sum_{-\infty}^{\infty} \beta_{n} z^{n}, \quad\left(r_{1}<z<r_{2}\right),
$$

is a second series then we find, on multiplying (25) and (27) that to the product

$$
c(z)=a(z) b(z)
$$

corresponds the series

$$
c(z)=\sum_{-\infty}^{\infty} \gamma_{n} z^{n}
$$

where

$$
\gamma_{n}=\sum_{n=-\infty}^{\infty} \alpha_{n-n} \beta_{\eta}
$$

Thus to the operation (28) of multiplication of the functions $a, b$, corresponds the operation of convolution (30) of the two sequences $\left\{\alpha_{n}\right\},\left\{\beta_{n}\right\}$, an operation which we write as

$$
\gamma=\alpha \beta \text {. }
$$

We mention incidentally a third interpretation of Laurent series isomorphic to the two already discussed. Indeed, consider the (4-way) infinite matrix

$$
\left\|\alpha_{j-i}\right\|
$$

in which $\alpha_{j-i}$ is the element in the $i$ th row and $j$ th column, both $i$ and $j$ assuming all integral values. Such matrices may be designated "striped" for the reason that all elements lying on a line, sloping down at a $45^{\circ}$ angle, are identical. To every sequence $\left\{\alpha_{n}\right\}$ corresponds one such matrix, and conversely. The isomorphism between such matrices and sequences becomes evident if we remark that the multiplication of two striped matrices $\left\|\alpha_{j-i}\right\|,\left\|\beta_{j-i}\right\|$, is another striped matrix $\left\|\gamma_{j-i}\right\|$, where the sequence $\left\{\gamma_{n}\right\}$ is given by (30). This remark throws some light on the connection between Laurent series and the case of finite matrices of $\$ 3$. 
We finally define the norm of the function $a(z)$, or of the sequence $\left\{\alpha_{n}\right\}$, as the non-negative number

$$
N(a)=N(\alpha)=\sum_{-\infty}^{\infty}\left|\alpha_{n}\right| .
$$

This norm also enjoys the two properties (22) or

$$
N(a+b) \leqq N(a)+N(b), \quad N(a b) \leqq N(a) N(b) .
$$

Their verification is immediate in this case.

We are now able to attack the general Problem of section 1. However, it is essential to discuss first the important case $m=1$ of reciprocation.

4.1. The reciprocation of Laurent series. With the norm of a Laurent series as defined by (33), the result of Hotelling and Lonseth (section 3) applies to Laurent series without any change. Assuming that the sum $a(z)$ of the given Laurent series (25) does not vanish in $R$, we are to find the expansion

Let

$$
w(z)=\frac{1}{a(z)}=\sum_{-\infty}^{\infty} \omega_{n} z^{n} .
$$

$$
w_{0}(\dot{z})=\sum_{-\infty}^{\infty} \omega_{n}^{(0)} z^{n}
$$

be an approximation to (35) such that

$$
N\left(1-a w_{0}\right)=N\left(I-\alpha \omega^{(0)}\right)=k<1 .
$$

The very important problem of how such approximations may be obtained will be discussed later (section 4.3). This starting sequence is now to be improved by the relation

$$
\omega^{(n+1)}=\omega^{(n)}+\omega^{(n)}\left(I-\alpha \omega^{(n)}\right) .
$$

The rapid convergence is assured by the Hotelling-Lonseth inequality

$$
N\left(\omega^{(n)}-\omega\right) \leqq N\left(\omega^{(0)}\right) \cdot k^{2 n} \cdot(1-k)^{-1} .
$$

Pending a discussion of procedures for obtaining the first approximation, we may therefore regard the numerical problem of reciprocation as solved. This implies that we may perform all four rational operations on Laurent series and that we may thus find the Laurent expansion of any rational function of Laurent series.

4.2. The general algebraic case. We turn now to the general case of the equation (4) with the two additional, and as we have seen, unessential restrictions that our ring $R$ contains the unit-circle $|z|=1$ and that the solution $w=w(z)$, of (4), be uniform in $R$. The problem is to find the numerical values of the coefficients of the expansion

$$
w(z)=\sum_{-\infty}^{\infty} \omega_{n} z^{n} .
$$

We return to our discussion (section 2) of the division-free Newton algorithm (11), especially in its expanded form (14). This discussion remains valid if applied to (4) rather than (1). The algorithm is in this case 


$$
w_{n+1}=w_{n}-f\left(w_{n}, z\right) \psi\left(w_{n}\right),
$$

the expanded form of which is

$$
w_{n+1}-w=b_{2}(w)\left(w_{n}-w\right)^{2}+\cdots+b_{2 m-1}(w)\left(w_{n}-w\right)^{2 m-1} .
$$

We have to remember, however, that $\psi\left(w_{n}\right)$ is a polynomial in $w_{n}$ with coefficients which are polynomials in $a_{v}$ divided by $D=D(z)$. Since $D$ is a polynomial in the $a_{v}$, we may first derive its Laurent expansion by additions and multiplications from the given Laurent series of the coefficients $a_{\nu}(z)$ of (4). Secondly, since

$$
D(z) \neq 0 \text { in } R,
$$

we may also find by the method of section 4.1 the Laurent expansion of $1 / D(z)$. In this way we arrive at the Laurent expansions of the coefficients of $\psi\left(w_{n}\right)$. These preliminary Laurent series operations allow to put the relation (41) in the form

$$
w_{n+1}=w_{n}+\left(c_{0}(z)+c_{1}(z) w_{n}+\cdots+c_{2 m-1}(z) w_{n}^{2 m-1}\right) ;
$$

where

$$
c_{\mu}(z)=\sum_{\nu} \gamma_{\mu \nu} z^{\nu}
$$

are numerically known Laurent expansions.

Now let

$$
w_{0}(z)=\sum_{-\infty}^{\infty} \omega_{\nu}^{(0)} z
$$

be an approximation to (40), (See section 4.3.) Starting from this approximation we obtain the successive series

$$
w_{n}(z)=\sum_{-\infty}^{\infty} \omega_{v}^{(n)} z^{n}
$$

by means of (43). This operation of deriving $w_{n+1}$ from $w_{n}$ is of course to be performed on the corresponding sequences of coefficients. By (43), (44), (46), the operation takes the form

$$
\omega^{(n+1)}=\omega^{(n)}+\left(\gamma_{0}+\gamma_{1} \omega^{(n)}+\cdots+\gamma_{2 m-1}\left(\omega^{(n)}\right)^{2 m-1}\right) .
$$

Will the expansion (46) converge towards the expansion (40) of the solution? To answer this question we return to the form (42) of our relation. Taking the norms of both sides of (42) and using the properties (34) of the norm, we obtain

$$
N\left(w_{n+1}-w\right) \leqq N\left(b_{2}(w)\right)\left[N\left(w_{n}-w\right)\right]^{2}+\cdots+N\left(b_{2 m-1}(w)\right)\left[N\left(w_{n}-w\right)\right]^{2 m-1} .
$$

This relation shows that if

$$
N\left(w_{0}-w\right)=\sum_{-\infty}^{\infty}\left|\omega_{\nu}^{(0)}-\omega_{\nu}\right|
$$

is sufficiently small then (48) will indeed imply

$$
\lim _{n \rightarrow \infty} N\left(w_{n}-w\right)=0
$$

with quadratic convergence. 
4.3. Derivation of an approximate Laurent expansion. The method for computation with Laurent series described in the previous sections will now become effective provided we can solve the following problem.

INITIAL APPROXIMATION PROBLEM. Let

$$
F(z)=\sum_{-\infty}^{\infty} c_{\nu} z^{\nu}
$$

be regular in the ring $R$ containing the circle $|z|=1$. This function $F(z)$, whose Laurent coefficients $c_{\nu}$ are unknown, is defined by an algebraic equation which allows us to compute the value of $F(z)$ for any given $z$ of $R$, in particular for any root of unity. We are to describe a practical method whereby, given $\epsilon>0$, we may compute the coefficients $c_{\nu}{ }^{*}$ of a Laurent series

$$
F^{*}(z)=\sum_{-\infty}^{\infty} c_{\nu}^{*} z^{\nu}
$$

regular in $R$, such that

$$
N\left(F-F^{*}\right)=\sum_{-\infty}^{\infty}\left|c_{\nu}-c_{\nu}^{*}\right|<\epsilon .
$$

We shall now solve this problem by the method of trigonometric interpolation. ${ }^{5}$ Let $m$ be a positive integer and let

$$
z_{\mu}=e^{2 \pi i \mu / m}, \quad(\mu=0,1, \cdots, m-1),
$$

be the $m$ th roots of unity. These roots of unity satisfy the following orthogonality relations

$$
\frac{1}{m} \sum_{\mu=0}^{m-1} z_{\mu}^{\prime} \bar{z}_{\mu}^{\prime}=\left\{\begin{array}{lll}
1 & \text { if } & \nu \equiv s(\bmod m) \\
0 & \text { if } & \nu \neq \equiv s(\bmod m) .
\end{array}\right.
$$

If $m$ is odd, $m=2 n+1$, we consider the Laurent polynomial

$$
F_{m}(z)=\sum_{\nu=-n}^{n} c_{m, \nu}
$$

having $m$ arbitrary coefficients.

If $m$ is even, $m=2 n$, we define our polynomial so as to contain again only $m$ arbitrary coefficients as

$$
F_{m}(z)=\sum_{\nu=-(n-1)}^{n-1} c_{m, \nu} z^{\nu}+\frac{1}{2} c_{m, n}\left(z^{n}+z^{-n}\right) .
$$

Whether $m$ is even or odd we may always write

$$
F_{m}(z)=\sum_{\nu=-n}^{n} c_{m, \nu} z^{\nu}, \quad\left(n=\left[\frac{m}{2}\right]\right),
$$

$\checkmark$ Concerning the subject of trigonometric interpolation we refer to the classical memoir by $\mathrm{Ch}$. J. de la Vallee Poussin, Sur la convergence des formules d'interpolation entre ordonées equidistantes, Bulletin de l'Academie royale de Belgique, 319-410 (1908), and to Dunham Jackson, The theory of approximation, American Mathematical Society Colloquium Publications, vol. 11, New York, 1930, chap. IV. 
where the summation symbol $\sum^{\prime}$ is to indicate that if $m$ is even, then

$$
c_{m,-n}=c_{m, n},
$$

and that the terms of (58) for $\nu= \pm n$ are to be taken with half their value. The relation $n=[m / 2]$ is to indicate that $n$ is the greatest integer not exceeding $m / 2$.

We shall now require the Laurent polynomial (58) to interpolate the function (51) in the points (54). This gives the $m$ equations

$$
\sum_{\nu=-n}^{n} c_{m, \nu} z_{\mu}^{\prime}=F\left(z_{\mu}\right), \quad(\mu=0,1, \cdots, m-1) .
$$

On multiplying (60) by $\bar{z}_{\mu}^{s} / m$ ( $s$ fixed, $-n \leqq s \leqq n$ ) we find in all cases, in view of (55) after summation by $\mu$, that

$$
c_{m, s}=\frac{1}{m} \sum_{\mu=0}^{m-1} F\left(z_{\mu}\right) \tilde{z}_{\mu}^{*}, \quad(-n \leqq s \leqq n) .
$$

The construction of our approximate Laurent expansion (58), i.e. (52), has now been completed. The following theorem will now show that the condition (53) may also be realized by the present method of construction.

THEOREM. We assume the Laurent series

$$
F(z)=\sum_{\nu=-\infty}^{\infty} c_{\nu} z^{\nu}
$$

to converge absolutely on the unit circle $|z|=1$, i.e.

$$
\sum_{-\infty}^{\infty}\left|c_{\nu}\right|<\infty .^{6}
$$

Then our interpolating Laurent polynomial (58) satisfies the condition

$$
\lim _{m \rightarrow \infty} N\left(F-F_{m}\right)=0 \text {. }
$$

Remark. Notice that the regularity of $F(x)$ in a ring containing $|z|=1$ implies our condition (63) but not conversely. This remark is of importance concerning calculations with absolutely convergent Fourier series. (See section 5.)

Proof. Let $N$ be a positive integer. We shall restrict ourselves to values of $m \geqq 2 N$, hence $n \geqq N$. We may then write

$$
N\left(F-F_{m}\right) \leqq \sum_{\nu=-N+1}^{N-1}\left|c_{\nu}-c_{m, \nu}\right|+\sum_{N \leqq|s| \leqq n}^{\prime}\left|c_{m, s}\right|+\sum_{|\nu| \geqq N}\left|c_{\nu}\right| .
$$

We shall now estimate the three sums on the right-hand side.

- See Dunham Jackson, loc. cit., for other conditions insuring the convergence of trigonometric interpolation. 
Let $\epsilon>0$ be given. In view of our assumption (63) we may choose $N$ such that

$$
\sum_{|\nu| \geqq N}\left|c_{\nu}\right|<\epsilon
$$

An upper bound for the second sum of (65) may now be obtained as follows. By (61) and (62) we have

$$
c_{m, s}=\frac{1}{m} \sum_{\mu=0}^{m-1} \bar{z}_{\mu}^{s} \sum_{\nu=-\infty}^{\infty} c_{\nu} z_{\mu}^{\nu}=\sum_{\nu=-\infty}^{\infty} c_{\nu} \frac{1}{m} \sum_{\mu=0}^{m-1} z_{\mu}^{\nu} \bar{z}_{\mu}^{s},
$$

and finally by (55)

$$
c_{m, s}=\sum_{\nu \equiv s(\bmod m)} c_{\nu}
$$

For all $m \geqq 2 N$, whether $m$ is even or odd, we now have

$$
\sum_{N \leqq|s| \leqq n}^{\prime}\left|c_{m, s}\right| \leqq \sum_{N \leqq|s| \leqq n} \sum_{\nu \equiv s(\bmod m)}\left|c_{\nu}\right| \leqq \sum_{|\nu| \geqq N}\left|c_{\nu}\right|<\epsilon
$$

by (66). We now return to (61). Since (62) converges uniformly on $|z|=1, F(z)$ is continuous on $|z|=1$ and (62) is its Fourier series. We therefore have the FourierCauchy relations

$$
c_{\nu}=\frac{1}{2 \pi i} \int_{|z|=1} F(z) z^{-n-1} d z .
$$

From the definition of this integral as a limit of Cauchy sums we may now write (with $z_{m}=1$ )

$$
\begin{aligned}
& c_{\nu}=\lim _{m \rightarrow \infty} \frac{1}{2 \pi i} \sum_{\mu=0}^{m-1} F\left(z_{\mu}\right) z_{\mu}^{-\nu-1}\left(z_{\mu+1}-z_{\mu}\right), \\
& c_{\nu}=\lim _{m \rightarrow \infty} \frac{1}{2 \pi i} \sum_{\mu=0}^{m-1} F\left(z_{\mu}\right) z_{\mu}^{-\nu}\left(e^{2 \pi i / m}-1\right),
\end{aligned}
$$

and finally, by (61),

$$
c_{\nu}=\lim _{m \rightarrow \infty} \frac{1}{m} \sum_{\mu=0}^{m-1} F\left(z_{\mu}\right) z_{\mu}^{-\nu}=\lim _{m \rightarrow \infty} c_{m \nu} .
$$

We now have indeed

$$
\sum_{\nu=-N+1}^{N-1}\left|c_{\nu}-c_{m, \nu}\right|<\epsilon, \text { provided } m>m_{0}(\epsilon) .
$$

By (65), (66), (68), and (71) we now have

$$
N\left(F-F_{m}\right)<3 \epsilon, \text { provided } m>m_{0}(\epsilon),
$$

and our theorem is established.

4.31. The 24-ordinate scheme of numerical harmonic analysis. The interpolation of our given function $F(z)$ in the 24 th roots of unity will provide satisfactory approxi- 
mation for most ordinary purposes. Let us assume for definiteness that $F(z)$ is real for real $z$. On the unit-circle $z=e^{i \theta}$ we then have

$$
F\left(e^{i \theta}\right)=R(\theta)+i I(\theta),
$$

where the real part $R(\theta)$ is an even function, while the imaginary part $I(\theta)$ is odd. Denote by

$$
F_{\mu}=R_{\mu}+i I_{\mu}, \quad(\mu=0,1, \cdots, 23),
$$

the computed values of our function at $15^{\circ}$-intervals in $\theta$, i.e. for the points (54) with $m=24$. We now interpolate the 13 ordinates $R_{\mu}(\mu=0,1, \cdots, 12)$ by a cosine polynomial

$$
A_{0}+A_{1} \cos \theta+\cdots+A_{11} \cos 11 \theta+A_{12} \cos 12 \theta,
$$

and the 13 ordinates $I_{0}=0, I_{1}, \cdots, I_{11}, I_{12}=0$ by a sine polynomial

$$
B_{1} \sin \theta+\cdots+B_{11} \sin 11 \theta \text {. }
$$

These polynomials are readily obtained by the 24-ordinate scheme as described in E. T. Whittaker and G. Robinson, The calculus of observations, ed. 3, 1940, section 137, pp. 273-278. The complex function (73) is now interpolated in the 24 points by the trigonometric polynomial

$$
\begin{aligned}
F_{24}\left(e^{i \theta}\right)= & A_{0}+A_{1} \cos \theta+\cdots+A_{11} \cos 11 \theta+A_{12} \cos 12 \theta \\
& +i B_{1} \sin \theta+\cdots+i B_{11} \sin 11 \theta
\end{aligned}
$$

Setting

$$
z=e^{i \theta}, \quad \cos \nu \theta=\frac{1}{2}\left(z^{\nu}+z^{-\nu}\right), \quad i \sin \nu \theta=\frac{1}{2}\left(z^{\nu}-z^{-\nu}\right),
$$

we obtain the Laurent sum with real coefficients

$$
\begin{aligned}
F_{24}(z)=A_{0} & +\sum_{\nu=1}^{11} \frac{1}{2}\left(A_{\nu}+B_{v}\right) z^{\nu}+\frac{1}{2} A_{12} z^{12} \\
& +\sum_{v=1}^{11} \frac{1}{2}\left(A_{\nu}-B_{v}\right) z^{-v}+\frac{1}{2} A_{12} z^{-12} .
\end{aligned}
$$

This initial approximate Laurent expansion will be used in section 6 in our example of reciprocation of a Laurent series.

5. Calculation with Fourier series. The method of calculation with Laurent series described in sections $4,4.1,4.2,4.3$ and 4.31 , applies unchanged to the realm of absolutely convergent Fourier series written in the complex form

$$
F(z)=\sum_{-\infty}^{\infty} c_{\nu} z^{\nu}, \text { where } z=e^{i \theta},
$$

with the definition of the norm as

$$
N(F)=\sum_{-\infty}^{\infty}\left|c_{\nu}\right|
$$

The general problem of section 1 may now be reformulated, replacing the ring $R$ by the unit circle $|z|=1$. The coefficients $a_{v}(z)$ of the equation (4) are now defined by 
given absolutely convergent Fourier series. The conditions (6) and (7) remain unchanged. The important fact that a uniform, continuous solution $w=w(z)$ of (4) along the unit-circle admits of an absolutely convergent Fourier expansion is now assured by a general theorem of $\mathrm{N}$. Wiener and P. Lévy. ${ }^{7}$ The effectiveness of the interpolation method of section 4.3 for obtaining a satisfactory initial approximate Fourier series is secured by our theorem of section 4.3 .

We finally mention briefly the special problem of the reciprocation of a nonvanishing absolutely convergent Fourier series

$$
A(\theta)=\frac{1}{2} a_{0}+\sum_{n=1}^{\infty}\left(a_{n} \cos n \theta+b_{n} \sin n \theta\right)
$$

with real coefficients $a_{v}, b_{v}$. In applying our method, we have to pass to the complex variable $z$, by means of the relations (77), obtaining the series

$$
A(\theta)=a(z)=\sum_{-\infty}^{\infty} \alpha_{n} z^{n},
$$

which is now to be reciprocated. The coefficients $\alpha_{n}$ being complex, it would appear that computations with complex numbers are unavoidable. This, however, is not the case since we may proceed as follows. Working with the real series

$$
f(z)=\sum_{-\infty}^{\infty} R\left(\alpha_{n}\right) z^{n}, \quad g(z)=\sum_{-\infty}^{\infty} I\left(\alpha_{n}\right) z^{n},
$$

we have by $(80)$

$$
\frac{1}{a(z)}=\frac{1}{f+i g}=\frac{f}{f^{2}+g^{2}}-i \frac{g}{f^{2}+g^{2}}=\sum_{-\infty}^{\infty} \omega_{n} z^{n} .
$$

Starting with (81) and operating with real series only, we now form the expansions of $f^{2}, g^{2}$ and then $f^{2}+g^{2}$. The real "Laurent" series of $f^{2}+g^{2}$ is now reciprocated by the method of section 4.1 and finally the series for

$$
f /\left(f^{2}+g^{2}\right), \quad g /\left(f^{2}+g^{2}\right)
$$

obtained by multiplications. This furnishes the complex values of the $\omega_{n}$ of (82). Returning to the variable $\theta$, by (77) we finally obtain the ordinary real Fourier expansion of

$$
1 / A(\theta)
$$

6. An example of reciprocation of a Laurent series. Our numerical example will benefit by the following general remark concerning the modified Newton algorithm (11). For simplicity we limit ourselves to the case of the equation (15) or

$$
a x^{m}-1=0
$$

which is solved by the recurrent relation

${ }^{7}$ See Antoni Zygmund, Trigonometrical series, Warszawa-Lw6w, 1935, pp. 140-142. 


$$
x_{n+1}=x_{n}+\frac{1}{m} x_{n}\left(1-a x_{n}^{m}\right) .
$$

Let us assume that our first approximation $x_{0}$ is of such accuracy that $x_{2}$ will have all the accuracy we want, while $x_{1}$ does not quite do. More precisely we assume the "residual"

$$
r=1-a x_{0}^{m}
$$

so small that we may neglect $r^{3}$ everywhere in our calculations. We may use this fact in eliminating $x_{1}$ between the two equations

$$
\begin{aligned}
& x_{1}=x_{0}+\frac{1}{m} x_{0}\left(1-x_{0}^{m}\right), \\
& x_{2}=x_{1}+\frac{1}{m} x_{1}\left(1-x_{1}^{m}\right) .
\end{aligned}
$$

Indeed, by (85), (86), we have

$$
x_{1}=x_{0}\left(1+\frac{1}{m} r\right)
$$

and neglecting $r^{3}$ we find

$$
x_{1}^{m}=x_{0}^{m}\left(1+r+\frac{m-1}{2 m} r^{2}\right) .
$$

If we then compute $x_{2}$ in this.way, i.e., neglecting $r^{3}$ wherever it appears, we easily find the following approximation to $x_{2}$ :

$$
x_{2}^{\prime}=x_{0}+\frac{1}{m} x_{0}\left(r+\frac{m+1}{2 m} r^{2}\right) .
$$

We may interpret both equations (85), (87) as a recurrence relation furnishing $x_{2}^{\prime}$ in terms of the first approximation $x_{0}$. This process converges "cubically." Indeed, a simple calculation will show that we may write (87) as

$$
x_{2}^{\prime}-x=\frac{(m+1)(2 m+1)}{6 x^{2}}\left(x_{0}-x\right)^{3}+(\text { terms of order }>3) .
$$

We note especially the following special case: To solve

$$
a x-1=0
$$

we set

$$
r=1-a x_{0}
$$

and compute

$$
x_{2}^{\prime}=x_{0}+x_{0}\left(r+r^{2}\right) .
$$

We turn now to our example which consists in expanding the reciprocal of the Bessel function

$$
J_{0}(z)=1-\frac{z^{2}}{2^{2}}+\frac{z^{4}}{(2 \cdot 4)^{2}}-\frac{z^{6}}{(2 \cdot 4 \cdot 6)^{2}}+\cdots
$$


into a Laurent series between the first two positive roots of this function, which are approximately $\xi_{1}=2.4, \xi_{2}=5.5$. In order to avoid even exponents we consider

$$
J_{0}(\sqrt{z})=1-\frac{z}{2^{2}}+\frac{z^{2}}{(2 \cdot 4)^{2}}-\cdots
$$

whose reciprocal is to be expanded in Laurent series between its zeros

$$
\xi_{1}^{2}=5.76 \text { and } \xi_{2}^{2}=30.25 \text {. }
$$

Let us notice that 13 is near the geometric mean of these numbers. In order to realize the condition (26) we replace in (92) $z$ by $13 z$, also changing the sign of the function for formal reasons. Thus let

$$
a(z)=-J_{0}(\sqrt{13 \bar{z}})=\sum_{n=0}^{\infty} \alpha_{n} z^{n}
$$

be the entire function whose reciprocal

$$
w(z)=-\frac{1}{J_{0}(\sqrt{13 z})}=\sum_{-\infty}^{\infty} \omega_{n} z^{n}
$$

we are to expand in a Laurent series convergent on and near the unit circle $|z|=1$. Below are the 10-place values of the coefficients $\alpha_{n}$ of (93) as computed by

$$
\alpha_{n}=(-1)^{n+1}(13) n /\left(2^{n} \cdot n !\right)^{2} .
$$

\begin{tabular}{|r|rr|}
\hline$n$ & \multicolumn{2}{|c|}{$\alpha_{n}$} \\
\hline 0 & -1.00000 & 00000 \\
1 & 3.25000 & 00000 \\
2 & -2.64062 & 50000 \\
3 & .95355 & 90278 \\
4 & -.19369 & 16775 \\
5 & .02517 & 99181 \\
6 & -.00227 & 31870 \\
7 & .00015 & 07726 \\
8 & -.00000 & 76564 \\
9 & .00000 & 03072 \\
10 & -.00000 & 00100 \\
11 & .00000 & 00003 \\
\hline$\sum$ & $=.39229$ & 24951 \\
\hline
\end{tabular}

\begin{tabular}{r|rr|rr|rr|rr|}
\hline$\mu$ & \multicolumn{2}{|c|}{$R_{\mu}$} & \multicolumn{2}{c|}{$I_{\mu}$} & \multicolumn{2}{|c|}{$A_{\mu}$} & \multicolumn{2}{|c|}{$B_{\mu}$} \\
\hline 0 & 2.549 & 122 & .000 & 000 & .601 & 975 & & \\
1 & 2.262 & 721 & -.257 & 032 & 1.063 & 727 & -.361 & 583 \\
2 & 1.655 & 481 & -.395 & 409 & .489 & 993 & -.144 & 072 \\
3 & 1.081 & 379 & -.427 & 381 & .219 & 762 & -.062 & 309 \\
4 & .661 & 333 & -.405 & 462 & .097 & 293 & -.028 & 189 \\
5 & .379 & 302 & -.361 & 625 & .042 & 831 & -.012 & 990 \\
6 & .193 & 500 & -.309 & 968 & .018 & 815 & -.006 & 018 \\
7 & .070 & 603 & -.256 & 308 & .008 & 261 & -.002 & 786 \\
8 & -.011 & 124 & -.202 & 973 & .003 & 630 & -.001 & 285 \\
9 & -.065 & 027 & -.150 & 752 & .001 & 604 & -.000 & 588 \\
10 & -.099 & 093 & -.099 & 722 & .000 & 727 & -.000 & 260 \\
11 & -.117 & 947 & -.049 & 615 & .000 & 368 & -.000 & 099 \\
12 & -.123 & 985 & .000 & 000 & .000 & 136 & & \\
\hline
\end{tabular}

From these values, rounded to 6 places, we computed to 6 places the values of $a\left(z_{\mu}\right)$ at the 24 th roots of unity

$$
z_{\mu}=\cos (15 \mu)^{0}+i \sin (15 \mu)^{0}, \quad(\mu=0,1, \cdots, 12),
$$

and from these the values of the reciprocal

$$
w\left(z_{\mu}\right)=1 / a\left(z_{\mu}\right)=R_{\mu}+i I_{\mu}, \quad(\mu=0,1, \cdots, 12),
$$


which are tabulated above. The coefficients $A_{\mu}$ and $B_{\mu}$ of the interpolating cosine and sine polynomials (75), (76) where then found by the 24 -ordinate scheme. They are tabulated above.

From these values we computed the coefficients $\omega_{n}^{(0)}$ of the approximation

$$
w_{0}(z)=\sum_{-12}^{12} \omega_{n}^{(0)} z^{n}
$$

according to (78) by the formulae

$$
\begin{aligned}
& \omega_{0}^{(0)}=A_{0}, \\
& \omega_{n}^{(0)}=\frac{1}{2}\left(A_{n}+B_{n}\right), \\
& \omega_{-n}^{(0)}=\frac{1}{2}\left(A_{n}-B_{n}\right), \\
& \omega_{12}^{(0)}=\omega_{-12}^{(0)}=\frac{1}{2} A_{12}, \quad(n=1,2, \cdots, 11) .
\end{aligned}
$$

These values rounded to 5 places are in the first column of the following table which contains the complete computation according to the relations (89) and (90). The last column headed $\omega=\omega^{(0)}+\omega^{(0)}\left(r+r^{2}\right)$ gives the 9-place values of the coefficients $\omega_{n}$ of (94).

Remarks. 1. The basic numerical process in this computation is obviously the convolution of sequences. Thus the second column $\alpha \omega^{(0)}$ is obtained by the convolution of the column $\alpha$ with the column $\omega^{(0)}$. According to the formula (30) this is done very simply rewriting the column $\alpha$, say, in reverse order, then matching it with the column $\omega^{(0)}$ such that the zero term of one column corresponds to the $n$th term of the other. The accumulated products of matching elements gives the $n$th term of the product column $\alpha \omega^{(0)}$. This operation is very familiar from the process of smoothing by means of a linear compound formula.

2. The operation of convolution of sequences implies an important check by means of their sums, for it is clear that the sum of the product column should equal the product of the sums of the factor sequence, except for the accumulated rounding error. At the very bottom of each column we wrote the actual sum of the sequence in that column. Directly below it we wrote (in parentheses) the value of this sum in terms of the sums of the columns which enter into its composition.

3 . The column of final residuals $I-\alpha \omega$ was also computed (values not recorded here) and its terms were found to be so small that a further repetition of the process, with our 10-place values of the $\alpha_{n}$, would not alter our 9-place values of the $\omega_{n}$. As final checks we found by (93)

$$
\begin{aligned}
a(1)^{-1} & =2.549118356, \quad a(-1)^{-1}=-.123985065, \\
a(\mathrm{i})^{-1} & =.193499936-.309967383 \mathrm{i} .
\end{aligned}
$$

The corresponding values of $w(z)$, computed by (94), were found to be

$$
\begin{aligned}
& w(1)=2.549118355, \quad w(-1)=-.123985067, \\
& w(\mathrm{i})=.193499940-.309967383 \mathrm{i} .
\end{aligned}
$$




\begin{tabular}{|c|c|c|c|c|c|}
\hline$n$ & $\omega^{(0)}$ & $\alpha \omega^{(0)}$ & $r=I-\alpha \omega^{(0)}$ & $r^{2}$ & \\
\hline $\begin{array}{r}-29 \\
-28 \\
-27 \\
-26 \\
-25 \\
-24 \\
-23 \\
-22 \\
-21 \\
-20 \\
-19 \\
-18 \\
-17 \\
-16 \\
-15 \\
-14 \\
-13 \\
-12 \\
-11 \\
-10 \\
-9 \\
-8 \\
-8 \\
-26 \\
-5 \\
-44 \\
-3 \\
-2 \\
-1 \\
0 \\
0 \\
1 \\
2 \\
3 \\
4 \\
5 \\
6 \\
7 \\
8 \\
9 \\
10 \\
11 \\
12 \\
13 \\
14 \\
15 \\
16 \\
17 \\
18 \\
19 \\
20 \\
21 \\
22 \\
23 \\
24 \\
25 \\
26 \\
27 \\
28 \\
29 \\
30 \\
31 \\
32\end{array}$ & $\begin{array}{l}.00007 \\
.00023 \\
.00049 \\
.00110 \\
.00246 \\
.00552 \\
.01242 \\
.02791 \\
.06274 \\
.14104 \\
.31703 \\
.71266 \\
.60198 \\
.35107 \\
.17296 \\
.07873 \\
.03455 \\
.01492 \\
.00640 \\
.00274 \\
.00117 \\
.00051 \\
.00023 \\
.00013 \\
.00007\end{array}$ & 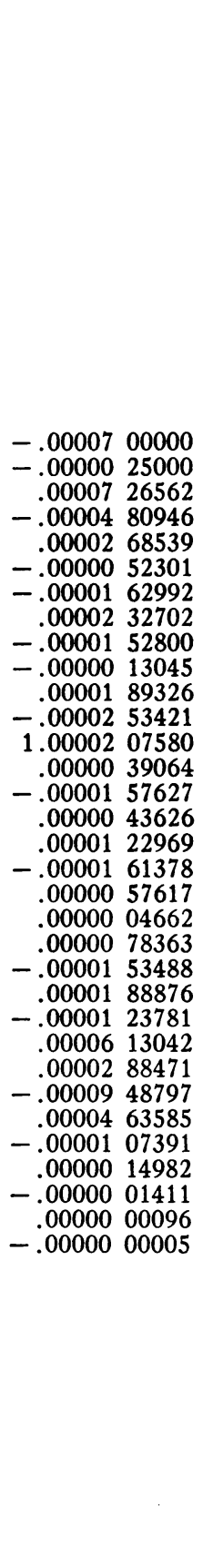 & 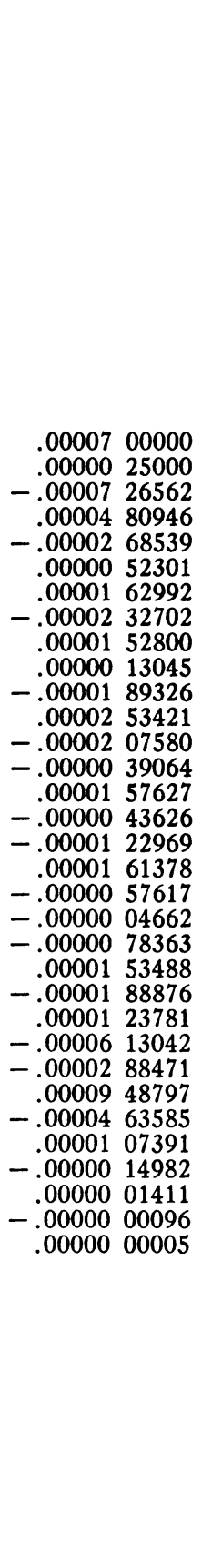 & $\begin{array}{c}-.00000 \\
.\end{array}$ & $\begin{array}{r}49 \\
4 \\
-102 \\
64 \\
18 \\
-64 \\
85 \\
-65 \\
9 \\
49 \\
-80 \\
62 \\
-\quad 7 \\
-68 \\
-97 \\
-42 \\
-35 \\
-61 \\
-27 \\
-17 \\
-24 \\
-14 \\
-13 \\
-13 \\
00056 \\
-70 \\
-237 \\
-89 \\
-116 \\
-174 \\
-145 \\
67 \\
30 \\
-91 \\
-91 \\
-37 \\
-29 \\
-81 \\
-75 \\
3 \\
-53 \\
-39 \\
-13 \\
-37 \\
-11 \\
-11 \\
0 \\
-32 \\
-21 \\
-124 \\
-53 \\
-92 \\
43 \\
3\end{array}$ \\
\hline$\sum$ & 2.54913 & $\begin{array}{c}1.0000045679 \\
(1.00000 \\
45680)\end{array}$ & -.0000045679 & $\begin{array}{l}.00000 \\
(.00000\end{array}$ & $\begin{array}{l}00002 \\
00000)\end{array}$ \\
\hline
\end{tabular}




\begin{tabular}{|c|c|c|c|}
\hline$n$ & $r+r^{2}$ & $\omega^{0}\left(r+r^{2}\right)$ & $\omega=\omega^{(0)}+\omega^{(0)}\left(r+r^{2}\right)$ \\
\hline $\begin{array}{r}-29 \\
-28 \\
-27 \\
-26 \\
-25 \\
-24 \\
-23 \\
-22 \\
-21 \\
-20 \\
-19 \\
-18 \\
-17 \\
-16 \\
-15 \\
-14 \\
-13 \\
-12 \\
-11 \\
-10 \\
-9 \\
-8 \\
-8 \\
-76 \\
-5 \\
-44 \\
-3 \\
-2 \\
-1 \\
0 \\
1 \\
2 \\
3 \\
4 \\
55 \\
6 \\
7 \\
89 \\
10 \\
11 \\
12 \\
13 \\
14 \\
15 \\
16 \\
17 \\
18 \\
19 \\
20 \\
21 \\
22 \\
23 \\
24 \\
25 \\
26 \\
27 \\
28 \\
29 \\
30 \\
31 \\
32\end{array}$ & 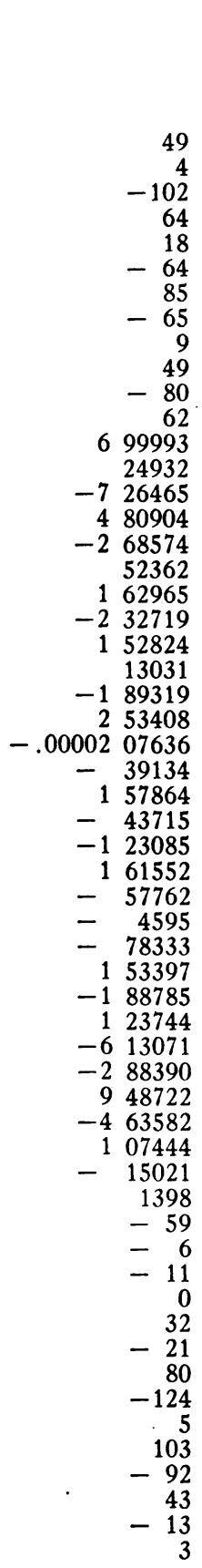 & 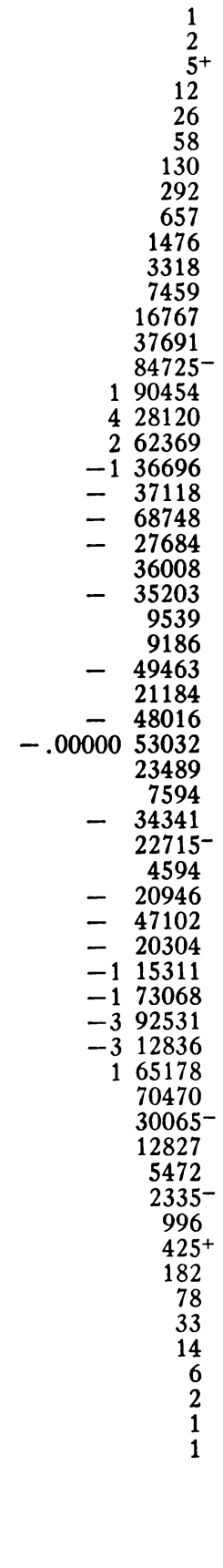 & 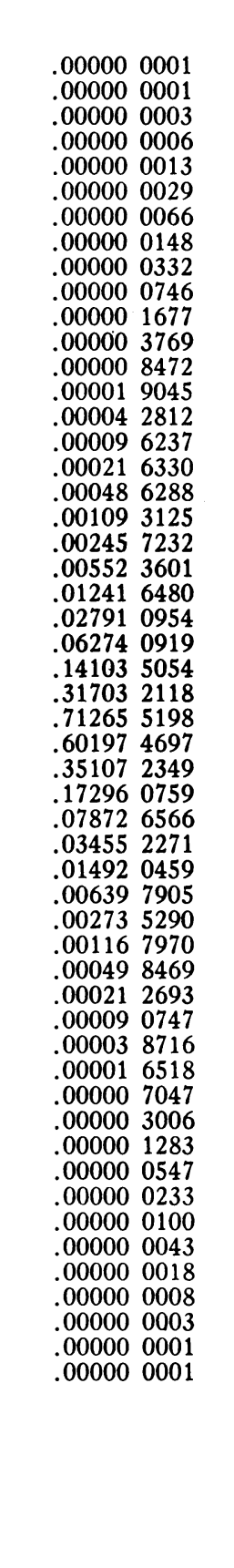 \\
\hline$\sum$ & -.0000045677 & $\left.\begin{array}{cc}-.00001 & 16443 \\
(-.00001 & 14637\end{array}\right)$ & 2.549118355 \\
\hline
\end{tabular}

